FACTA UNIVERSITATIS (NIŠ)

Ser. Math. Inform. Vol. 35, No 2 (2020), 295-310

https://doi.org/10.22190/FUMI2002295S

\title{
$\eta$-RICCI SOLITONS ON KENMOTSU MANIFOLD WITH GENERALIZED SYMMETRIC METRIC CONNECTION
}

\author{
Mohd Danish Siddiqi and Ŏguzhan Bahadır
}

(C) 2020 by University of Niš, Serbia | Creative Commons Licence: CC BY-NC-ND

\begin{abstract}
The objective of the present paper is to study the $\eta$-Ricci solitons on Kenmotsu manifold with generalized symmetric metric connection of type $(\alpha, \beta)$. Ricci and $\eta$-Ricci solitons with generalized symmetric metric connection of type $(\alpha, \beta)$ have been discussed, satisfying the conditions $\bar{R} \cdot \bar{S}=0, \bar{S} \cdot \bar{R}=0, \bar{W}_{2} \cdot \bar{S}=0$ and $\bar{S} \cdot \bar{W}_{2}=0$.. Finally, we have constructed an example of Kenmotsu manifold with generalized symmetric metric connection of type $(\alpha, \beta)$ admitting $\eta$-Ricci solitons.

Keywords: Kenmotsu manifold; Generalized symmetric metric connection; $\eta$-Ricci soliton; Ricci soliton, Einstein manifold.
\end{abstract}

\section{Introduction}

A linear connection $\bar{\nabla}$ is said to be generalized symmetric connection if its torsion tensor $T$ is of the form

$$
T(X, Y)=\alpha\{u(Y) X-u(X) Y\}+\beta\{u(Y) \varphi X-u(X) \varphi Y\},
$$

for any vector fields $X, Y$ on a manifold, where $\alpha$ and $\beta$ are smooth functions. $\varphi$ is a tensor of type $(1,1)$ and $u$ is a 1 -form associated with a non-vanishing smooth non-null unit vector field $\xi$. Moreover, the connection $\bar{\nabla}$ is said to be a generalized symmetric metric connection if there is a Riemannian metric $g$ in $M$ such that $\bar{\nabla} g=0$, otherwise it is non-metric.

In the equation (1.1), if $\alpha=0(\beta=0)$, then the generalized symmetric connection is called $\beta$ - quarter-symmetric connection $(\alpha-$ semi-symmetric connection), respectively. Moreover, if we choose $(\alpha, \beta)=(1,0)$ and $(\alpha, \beta)=(0,1)$, then the generalized symmetric connection is reduced to a semi-symmetric connection and quarter-symmetric connection, respectively. Therefore, a generalized symmetric

Received September 03, 2018; accepted January 01, 2019

2010 Mathematics Subject Classification. 53C05, 53D15, 53C25. 
connections can be viewed as a generalization of semi-symmetric connection and quarter-symmetric connection. These two connections are important for both the geometry study and applications to physics. In [12], H. A. Hayden introduced a metric connection with non-zero torsion on a Riemannian manifold. The properties of Riemannian manifolds with semi-symmetric (symmetric) and non-metric connection have been studied by many authors (see [1], [9], [10] , [24], [26]). The idea of quarter-symmetric linear connections in a differential manifold was introduced by S.Golab [11]. In [23], Sharfuddin and Hussian defined a semi-symmetric metric connection in an almost contact manifold, by setting

$$
T(X, Y)=\eta(Y) X-\eta(X) Y .
$$

In [13], [25] and [19] the authors studied the semi-symmetric metric connection and semi-symmetric non-metric connection in a Kenmotsu manifold, respectively.

In the present paper, we have defined new connection for Kenmotsu manifold, generalized symmetric metric connection. This connection is the generalized form of semi-symmetric metric connection and quarter-symmetric metric connection.

On the other hand, a Ricci soliton is a natural generalization of an Einstein metric. In 1982, R. S. Hamilton [14] said that the Ricci solitons moved under the Ricci flow simply by diffeomorphisms of the initial metric, that is, they are sationary points of the Ricci flow:

$$
\frac{\partial g}{\partial t}=-2 \operatorname{Ric}(g)
$$

Definition 1.1. A Ricci soliton $(g, V, \lambda)$ on a Riemannian manifold is defined by

$$
\mathcal{L}_{V} g+2 S+2 \lambda=0
$$

where $S$ is the Ricci tensor, $\mathcal{L}_{V}$ is the Lie derivative along the vector field $V$ on $M$ and $\lambda$ is a real scalar. Ricci soliton is said to be shrinking, steady or expanding according as $\lambda<0, \lambda=0$ and $\lambda>0$, respectively.

In 1925, H. Levy [16] in Theorem 4, proved that a second order parallel symmetric non-singular tensor in real space forms is proportional to the metric tensor. Later, R. Sharma [22] initiated the study of Ricci solitons in contact Riemannian geometry. After that, Tripathi [28], Nagaraja et. al. [17] and others like C. S. Bagewadi et. al. [4] extensively studied Ricci solitons in almost contact metric manifolds. In 2009, J. T. Cho and M. Kimura [6] introduced the notion of $\eta$-Ricci solitons and gave a classification of real hypersurfaces in non-flat complex space forms admitting $\eta$-Ricci solitons. $\eta$ - Ricci solitons in almost paracontact metric manifolds have been studied by A. M. Blaga et. al. [2]. A. M. Blaga and various others authors have also studied $\eta$-Ricci solitons in manifolds with different structures (see [3], [20]). It is natural and interesting to study $\eta$-Ricci solitons in almost contact metric manifolds with this new connection.

Therefore, motivated by the above studies, in this paper we will study the $\eta$-Ricci solitons in a Kenmotsu manifold with respect to a generalized symmetric metric 
connection. We shall consider $\eta$-Ricci solitons in the almost contact geometry, precisely, on an Kenmotsu manifold with generalized symmetric metric connection which satisfies certain curvature properties: $\bar{R} \cdot \bar{S}=0, \bar{S} \cdot \bar{R}=0, W_{2} \cdot \bar{S}=0$ and $\bar{S} . \bar{W}_{2}=0$ respectively.

\section{Preliminaries}

A differentiable $M$ manifold of dimension $n=2 m+1$ is called almost contact metric manifold [5], if it admits a $(1,1)$ tensor field $\phi$, a contravaryant vector field $\xi$, a $1-$ form $\eta$ and Riemannian metric $g$ which satisfies

$$
\begin{aligned}
\phi \xi & =0 \\
\eta(\phi X) & =0 \\
\eta(\xi) & =1 \\
\phi^{2}(X) & =-X+\eta(X) \xi, \\
g(\phi X, \phi Y) & =g(X, Y)-\eta(X) \eta(Y), \\
g(X, \xi) & =\eta(X),
\end{aligned}
$$

for all vector fields $X, Y$ on $M$. If we write $g(X, \phi Y)=\Phi(X, Y)$, then the tensor field $\phi$ is a anti-symmetric $(0,2)$ tensor field [5]. If an almost contact metric manifold satisfies

$$
\begin{aligned}
\left(\nabla_{X} \phi\right) Y & =g(\phi X, Y) \xi-\eta(Y) \phi X \\
\nabla_{X} \xi & =X-\eta(X) \xi
\end{aligned}
$$

then $M$ is called a Kenmotsu manifold, where $\nabla$ is the Levi-Civita connection of $g$ [18].

In Kenmotsu manifolds the following relations hold [18]:

$$
\begin{aligned}
\left(\nabla_{X} \eta\right) Y & =g(\phi X, \phi Y) \\
g(R(X, Y) Z, \xi) & =\eta(R(X, Y) Z)=g(X, Z) \eta(Y)-g(Y, Z) \eta(X) \\
R(\xi, X) Y & =\eta(Y) X-g(X, Y) \xi \\
R(X, Y) \xi & =\eta(X) Y-\eta(Y) X \\
R(\xi, X) \xi & =X-\eta(X) \xi \\
S(X, \xi) & =-(n-1) \eta(X), \\
S(\phi X, \phi Y) & =S(X, Y)+(n-1) \eta(X) \eta(Y)
\end{aligned}
$$

for any vector fields $X, Y$ and $Z$, where $R$ and $S$ are the the curvature and Ricci the tensors of $M$, respectively.

A Kenmotsu manifold $M$ is said to be generalized $\eta$ Einstein if its Ricci tensor $\mathrm{S}$ is of the form

$$
S(X, Y)=a g(X, Y)+b \eta(X) \eta(Y)+c g(\phi X, Y),
$$

for any $X, Y \in \Gamma(T M)$, where $a, b$ and $c$ are scalar functions such that $b \neq 0$ and $c \neq 0$. If $c=0$ then $M$ is called $\eta$ Einstein manifold. 


\section{Generalized Symmetric Metric Connection in a Kenmotsu Manifold}

Let $\bar{\nabla}$ be a linear connection and $\nabla$ be a Levi-Civita connection of an almost contact metric manifold $M$ such that

$$
\bar{\nabla}_{X} Y=\nabla_{X} Y+H(X, Y),
$$

for any vector field $X$ and $Y$. Where $H$ is a tensor of type $(1,2)$. For $\bar{\nabla}$ to be a generalized symmetric metric connection of $\nabla$, we have

$$
H(X, Y)=\frac{1}{2}\left[T(X, Y)+T^{\prime}(X, Y)+T^{\prime}(Y, X)\right],
$$

where $T$ is the torsion tensor of $\bar{\nabla}$ and

$$
g\left(T^{\prime}(X, Y), Z\right)=g(T(Z, X), Y) .
$$

From (1.1) and (3.3) we get

$$
T^{\prime}(X, Y)=\alpha\{\eta(X) Y-g(X, Y) \xi\}+\beta\{-\eta(X) \phi Y-g(\phi X, Y) \xi\} .
$$

Using (1.1), (3.2) and (3.4) we obtain

$$
H(X, Y)=\alpha\{\eta(Y) X-g(X, Y) \xi\}+\beta\{-\eta(X) \phi Y\} .
$$

Corollary 3.1. For a Kenmotsu manifold, generalized symmetric metric connection $\bar{\nabla}$ is given by

$$
\bar{\nabla}_{X} Y=\nabla_{X} Y+\alpha\{\eta(Y) X-g(X, Y) \xi\}-\beta \eta(X) \phi Y .
$$

If we choose $(\alpha, \beta)=(1,0)$ and $(\alpha, \beta)=(0,1)$, generalized metric connection is reduced to a semi-symmetric metric connection and quarter-symmetric metric connection as follows:

$$
\begin{gathered}
\bar{\nabla}_{X} Y=\nabla_{X} Y+\eta(Y) X-g(X, Y) \xi \\
\bar{\nabla}_{X} Y=\nabla_{X} Y-\eta(X) \phi Y .
\end{gathered}
$$

From (3.6) we have the following proposition

Proposition 3.1. Let $M$ be a Kenmotsu manifold with generalized metric connection. We have the following relations:

$$
\begin{aligned}
\left(\bar{\nabla}_{X} \phi\right) Y & =(\alpha+1)\{g(\phi X, Y) \xi-\eta(Y) \phi X\} \\
\bar{\nabla}_{X} \xi & =(\alpha+1)\{X-\eta(X) \xi\} \\
\left(\bar{\nabla}_{X} \eta\right) Y & =(\alpha+1)\{g(X, Y)-\eta(Y) \eta(X)\}
\end{aligned}
$$

for any $X, Y, Z \in \Gamma(T M)$. 


\section{Curvature Tensor on Kenmotsu manifold with generalized symmetric metric connection}

Let $M$ be an $n$-dimensional Kenmotsu manifold. The curvature tensor $\bar{R}$ of the generalized metric connection $\bar{\nabla}$ on $M$ is defined by

$$
\bar{R}(X, Y) Z=\bar{\nabla}_{X} \bar{\nabla}_{Y} Z-\bar{\nabla}_{Y} \bar{\nabla}_{X} Z-\bar{\nabla}_{[X, Y]} Z
$$

Using the proposition 3.1, from (3.6) and (4.1) we have

$$
\begin{aligned}
(4.2) \bar{R}(X, Y) Z= & R(X, Y) Z+\left\{\left(-\alpha^{2}-2 \alpha\right) g(Y, Z)+\left(\alpha^{2}+a\right) \eta(Y) \eta(Z)\right\} X \\
+ & \left\{\left(\alpha^{2}+2 \alpha\right) g(X, Z)+\left(-\alpha^{2}-\alpha\right) \eta(X) \eta(Z)\right\} Y \\
+ & \left\{\left(\alpha^{2}+\alpha\right)[g(Y, Z) \eta(X)-g(X, Z) \eta(Y)]\right. \\
+ & (\beta+\alpha \beta)[g(X, \phi Z) \eta(Y)-g(Y, \phi Z) \eta(X)]\} \xi \\
+ & (\beta+\alpha \beta) \eta(Y) \eta(Z) \phi X-(\beta+\alpha \beta) \eta(X) \eta(Z) \phi Y
\end{aligned}
$$

where

$$
R(X, Y) Z=\nabla_{X} \nabla_{Y} Z-\nabla_{Y} \nabla_{X} Z-\nabla_{[X, Y]} Z
$$

is the curvature tensor with respect to the Levi-Civita connection $\nabla$.

Using (2.10), (2.11), (2.12), (2.13) and (4.2) we give the following proposition:

Proposition 4.1. Let $M$ be an $n$ - dimensional Kenmotsu manifold with generalized symmetric metric connection of type $(\alpha, \beta)$. Then we have the following equations:

$$
\begin{gathered}
\bar{R}(X, Y) \xi=(\alpha+1)\{\eta(X) Y-\eta(Y) X+\beta[\eta(Y) \phi X-\eta(X) \phi Y]\} \\
\bar{R}(\xi, X) Y=(\alpha+1)\{\eta(Y) X-g(X, Y) \xi+\beta[\eta(Y) \phi X-g(X, \phi Y) \xi]\} \\
\bar{R}(\xi, Y) \xi=(\alpha+1)\{Y-\eta(Y) \xi-\beta \phi Y\} \\
\eta(\bar{R}(X, Y) Z=(\alpha+1)\{\eta(Y) g(X, Z)-\eta(X) g(Y, Z) \\
+\beta[\eta(Y) g(X, \phi Z)-\eta(X) g(Y, \phi Z)]\}
\end{gathered}
$$

for any $X, Y, Z \in \Gamma(T M)$.

We know that Ricci tensor is defined by

$$
\bar{S}(Y, Z)=\sum_{i=1}^{n} g\left(\bar{R}\left(e_{i}, Y\right) Z, e_{i}\right)
$$


where $Y, Z \in \Gamma(T M),\left\{e_{1}, e_{2}, \ldots, e_{n}\right\}$ is viewed as orthonormal frame. We can calculate the Ricci tensor with respect to generalized symmetric metric connection as follows:

$$
\begin{aligned}
\bar{S}(Y, Z)= & S(Y, Z)+\left\{(2-n) \alpha^{2}+(3-2 n) \alpha\right\} g(Y, Z)+(n-2)\left(\alpha^{2}+\alpha\right) \eta(Y) \eta(Z) \\
(4.8)= & -(\beta+\alpha \beta) g(Y, \phi Z),
\end{aligned}
$$

where $S$ is Ricci tensor with respect to Levi-Civita connection.

Example 4.1. We consider a 3-dimensional manifold $M=\left\{(x, y, z) \in R^{3}: x \neq 0\right\}$, where $(x, y, z)$ are the standard coordinates in $R^{3}$. Let $E_{1}, E_{2}, E_{3}$ be a linearly independent global frame on $M$ given by

$$
E_{1}=x \frac{\partial}{\partial z}, E_{2}=x \frac{\partial}{\partial y}, E_{3}=-x \frac{\partial}{\partial x} .
$$

Let $\mathrm{g}$ be the Riemannian metric defined by

$$
g\left(E_{1}, E_{2}\right)=g\left(E_{1}, E_{3}\right)=g\left(E_{2}, E_{3}\right)=0, g\left(E_{1}, E_{1}\right)=g\left(E_{2}, E_{2}\right)=g\left(E_{3}, E_{3}\right)=1,
$$

Let $\eta$ be the 1-form defined by $\eta(U)=g\left(U, E_{3}\right)$, for any $U \in T M$. Let $\phi$ be the $(1,1)$ tensor field defined by $\phi E_{1}=E_{2}, \phi E_{2}=-E_{1}$ and $\phi E_{3}=0$. Then, using the linearity of $\phi$ and $g$ we have $\eta\left(E_{3}\right)=1, \phi^{2} U=-U+\eta(U) E_{3}$ and $g(\phi U, \phi W)=g(U, W)-\eta(U) \eta(W)$ for any $U, W \in T M$. Thus for $E_{3}=\xi,(\phi, \xi, \eta, g)$ an almost contact metric manifold is defined.

Let $\nabla$ be the Levi-Civita connection with respect to the Riemannian metric $g$. Then we have

$$
\left[E_{1}, E_{2}\right]=0, \quad\left[E_{1}, E_{3}\right]=E_{1}, \quad\left[E_{2}, E_{3}\right]=E_{2},
$$

Using Koszul formula for the Riemannian metric $g$, we can easily calculate

$$
\begin{array}{ccc}
\nabla_{E_{1}} E_{1}=-E_{3}, & \nabla_{E_{1}} E_{2}=0 . & \nabla_{E_{1}} E_{3}=E_{1}, \\
\nabla_{E_{2}} E_{1}=0, & \nabla_{E_{2}} E_{2}=-E_{3}, & \nabla_{E_{2}} E_{3}=0, \\
\nabla_{E_{3}} E_{1}=0, & \nabla_{E_{3}} E_{2}=0, & \nabla_{E_{3}} E_{3}=0 .
\end{array}
$$

From the above relations, it can be easily seen that

$\left(\nabla_{X} \phi\right) Y=g(\phi X, Y) \xi-\eta(Y) \phi X, \quad \nabla_{X} \xi=X-\eta(X) \xi$, for all $E_{3}=\xi$. Thus the manifold $M$ is a Kenmotsu manifold with the structure $(\phi, \xi, \eta, g)$. for $\xi=E_{3}$. Therefore, the manifold $M$ under consideration is a Kenmotsu manifold of dimension three.

\section{Ricci and $\eta$-Ricci solitons on $(M, \phi, \xi, \eta, g$,}

Let $(M, \phi, \xi, \eta, g$,$) be an almost contact metric manifold. Consider the equation$

$$
\mathcal{L}_{\xi} g+2 \bar{S}+2 \lambda+2 \mu \eta \otimes \eta=0
$$


where $\mathcal{L}_{\xi}$ is the Lie derivative operator along the vector field $\xi, \bar{S}$ is the Ricci curvature tensor field with respect to the generalized symmetric metric connection of the metric $g$, and $\lambda$ and $\mu$ are real constants. Writing $\mathcal{L}_{\xi}$ in terms of the generalized symmetric metric connection $\bar{\nabla}$, we obtain:

$$
2 \bar{S}(X, Y)=-g\left(\bar{\nabla}_{X} \xi, Y\right)-g\left(X, \bar{\nabla}_{Y} \xi\right)-2 \lambda g(X, Y)-2 \mu \eta(X) \eta(Y),
$$

for any $X, Y \in \chi(M)$.

The data $(g, \xi, \lambda, \mu)$ which satisfy the equation (4.9) is said to be an $\eta$-Ricci soliton on $M$ [10]. In particular, if $\mu=0$ then $(g, \xi, \lambda)$ is called Ricci soliton [6] and it is called shrinking, steady or expanding, according as $\lambda$ is negative, zero or positive respectively [6].

Here is an example of $\eta$-Ricci soliton on Kenmotsu manifold with generalized symmetric metric connection.

Example 5.1. Let $M(\phi, \xi, \eta, g)$ be the Kenmotsu manifold considered in example 4.3 .

Let $\bar{\nabla}$ be a generalized symmetric metric connection, we obtain: Using the above relations, we can calculate the non-vanishing components of the curvature tensor as follows:

$$
\begin{array}{r}
R\left(E_{1}, E_{2}\right) E_{1}=E_{2}, R\left(E_{1}, E_{2}\right) E_{2}=-E_{1}, R\left(E_{1}, E_{3}\right) E_{1}=E_{3} \\
R\left(E_{1}, E_{3}\right) E_{3}=-E_{1}, R\left(E_{2}, E_{3}\right) E_{2}=E_{3}, R\left(E_{2}, E_{3}\right) E_{3}=-E_{2}
\end{array}
$$

From the equations (5.3) we can easily calculate the non-vanishing components of the Ricci tensor as follows:

$$
S\left(E_{1}, E_{1}\right)=-2, S\left(E_{2}, E_{2}\right)=-2, S\left(E_{3}, E_{3}\right)=-2
$$

Now, we can make similar calculations for generalized metric connection. Using (3.6) in the above equations, we get

$$
\begin{array}{rcc}
\bar{\nabla}_{E_{1}} E_{1}=-(1+\alpha) E_{3}, & \bar{\nabla}_{E_{1}} E_{2}=0 . & \bar{\nabla}_{E_{1}} E_{3}=(1+\alpha) E_{1}, \\
\bar{\nabla}_{E_{2}} E_{1}=0, & \bar{\nabla}_{E_{2}} E_{2}=-(1+\alpha) E_{3}, & \bar{\nabla}_{E_{2}} E_{3}=\alpha E_{2}, \\
\bar{\nabla}_{E_{3}} E_{1}=-\beta E_{2}, & \bar{\nabla}_{E_{3}} E_{2}=\beta E_{1}, & \bar{\nabla}_{E_{3}} E_{3}=0 .
\end{array}
$$

From (5.5), we can calculate the non-vanishing components of curvature tensor with respect to generalized metric connection as follows:

$$
\begin{array}{rc}
\bar{R}\left(E_{1}, E_{2}\right) E_{1}=(1+\alpha)^{2} E_{2}, & \bar{R}\left(E_{1}, E_{2}\right) E_{2}=-(1+\alpha)^{2} E_{1}, \\
\bar{R}\left(E_{1}, E_{3}\right) E_{1}=(1+\alpha) E_{3} & \bar{R}\left(E_{1}, E_{3}\right) E_{3}=(1+\alpha)\left(\beta E_{2}-E_{1}\right), \\
\bar{R}\left(E_{2}, E_{3}\right) E_{2}=(1+\alpha) E_{3}, & \bar{R}\left(E_{2}, E_{3}\right) E_{3}=-(1+\alpha)\left(-\beta E_{1}+E_{2}\right) \\
\bar{R}\left(E_{3}, E_{2}\right) E_{1}=-(1+\alpha) \beta E_{3}, & \bar{R}\left(E_{3}, E_{1}\right) E_{2}=(1+\alpha) \beta E_{3}, .
\end{array}
$$


From (5.6), the non-vanishing components of the Ricci tensor are as follows:

$$
\begin{gathered}
\bar{S}\left(E_{1}, E_{1}\right)=-(1+\alpha)(2+\alpha), \quad \bar{S}\left(E_{2}, E_{2}\right)=-(1+\alpha)(2+\alpha), \\
\bar{S}\left(E_{3}, E_{3}\right)=-2(1+\alpha) .
\end{gathered}
$$

From (5.2) and (5.5) we get

$(5.8) 2(1+\alpha)\left[g\left(e_{i}, e_{i}\right)-\eta\left(e_{i}\right) \eta\left(e_{i}\right)\right]+2 \bar{S}\left(e_{i}, e_{i}\right)+2 \lambda g\left(e_{i}, e_{i}\right)+2 \mu \eta\left(e_{i}\right) \eta\left(e_{i}\right)=0$

for all $i \in\{1,2,3\}$, and we have $\lambda=(1+\alpha)^{2}$ (i.e. $\left.\lambda>0\right)$ and $\mu=1-\alpha^{2}$, the data $(g, \xi, \lambda, \mu)$ is an $\eta$-Ricci soliton on $(M, \phi, \xi, \eta, g)$. If $\alpha=-1$ which is steady and if $\alpha \neq-1$ which is expanding.

\section{Parallel symmetric second order tensors and $\eta$-Ricci solitons in}

\section{Kenmotsu manifolds}

An important geometrical object in studying Ricci solitons is well known to be a symmetric $(0,2)$-tensor field which is parallel with respect to the generalized symmetric metric connection.

Now, let fix $h$ a symmetric tensor field of (0,2)-type which we suppose to be parallel with respect to generalized symmetric metric connection $\bar{\nabla}$ that is $\bar{\nabla} h=0$. By applying Ricci identity [7]

$$
\bar{\nabla}^{2} h(X, Y ; Z, W)-\bar{\nabla}^{2} h(X, Y ; Z, W)=0,
$$

we obtain the relation

$$
h(\bar{R}(X, Y) Z, W)+h(Z, \bar{R}(X, Y) W)=0 .
$$

Replacing $Z=W=\xi$ in (6.2) and by using (4.4) and by the symmetry of $h$ it follows $h(\bar{R}(X, Y) \xi, \xi)=0$ for any $X, Y \in \chi(M)$ and

$$
\begin{gathered}
(\alpha+1) \eta(X) h(Y, \xi)-(\alpha+1) \eta(Y) h(X, \xi) \\
+(\alpha+1) \eta(X) h(\xi, Y)-(\alpha+1) \eta(Y) h(\xi, X)
\end{gathered}
$$

$(6.5)+\beta \eta(Y) h(\phi X, \xi)-\beta \eta(X) h(\phi Y, \xi)+\beta \eta(Y) h(\xi, \phi X)-\beta \eta(X) h(\xi, \phi Y)=0$

Putting $X=\xi$ in (6.3) and by the virtue of (2.4), we obtain

$$
2(\alpha+1)[h(Y, \xi)-\eta(Y) h(\xi, \xi)]-2 \beta h(\phi Y, \xi)=0 .
$$

or

$$
2(\alpha+1)[h(Y, \xi)-g(Y, \xi) h(\xi, \xi)]-2 \beta(\phi Y, \xi)=0 .
$$

Suppose $(\alpha+1) \neq 0, \beta=0$ it results

$$
h(Y, \xi)-\eta(Y) h(\xi, \xi)=0
$$


for any $Y \in \chi(M)$, equivalent to

$$
h(Y, \xi)-g(Y, \xi) h(\xi, \xi)=0,
$$

for any $Y \in \chi(M)$. Differentiating the equation (6.9) covariantly with respect to the vector field $X \in \chi(M)$, we obtain

$$
h\left(\bar{\nabla}_{X} Y, \xi\right)+h\left(Y, \bar{\nabla}_{X} \xi\right)=h(\xi, \xi)\left[g\left(\bar{\nabla}_{X} Y, \xi\right)+g\left(Y, \bar{\nabla}_{X} \xi\right)\right] .
$$

Using (4.4) in (6.10), we obtain

$$
h(X, Y)=h(\xi, \xi) g(X, Y),
$$

for any $X, Y \in \chi(M)$. The above equation gives the conclusion:

Theorem 6.1. Let $(M, \phi, \xi, \eta, g$,$) be a Kenmotsu manifold with generalized sym-$ metric metric connection also with non-vanishing $\xi$-sectional curvature and endowed with a tensor field of type $(0,2)$ which is symmetric and $\phi$-skew-symmetric. If $h$ is parallel with respect to $\bar{\nabla}$, then it is a constant multiple of the metric tensor $g$.

On a Kenmotsu manifold with generalized symmetric metric connection using equation (3.10) and $\mathcal{L}_{\xi} g=2(g-\eta \otimes \eta)$, the equation (5.2) becomes:

$$
\bar{S}(X, Y)=-(\lambda+\alpha+1) g(X, Y)+(\alpha+1-\mu) \eta(X) \eta(Y) .
$$

In particular, $X=\xi$, we obtain

$$
\bar{S}(X, \xi)=-(\lambda+\mu) \eta(X) .
$$

In this case, the Ricci operator $\bar{Q}$ defined by $g(\bar{Q} X, Y)=\bar{S}(X, Y)$ has the expression

$$
\bar{Q} X=-(\lambda+\alpha+1) X+(\alpha+1-\mu) \eta(X) \eta(X) \xi .
$$

Remark that on a Kenmostu manifold with generalized symmetric metric connection, the existence of an $\eta$-Ricci soliton implies that the characteristic vector field $\xi$ is an eigenvector of Ricci operator corresponding to the eigenvalue $-(\lambda+\mu)$.

Now we shall apply the previous results on $\eta$-Ricci solitons.

Theorem 6.2. Let $(M, \phi, \xi, \eta, g)$ be a Kenmotsu manifold with generalized symmetric metric connection. Assume that the symmetric $(0,2)$-tensor filed $h=\mathcal{L}_{\xi} g+$ $2 S+2 \mu \eta \otimes \eta$ is parallel with respect to the generalized symmetric metric connection associated to $g$. Then $\left(g, \xi,-\frac{1}{2} h(\xi, \xi), \mu\right)$ yields an $\eta$-Ricci soliton.

Proof. Now, we can calculate

$$
h(\xi, \xi)=\mathcal{L}_{\xi} g(\xi, \xi)+2 \bar{S}(\xi, \xi)+2 \mu \eta(\xi) \eta(\xi)=-2 \lambda,
$$

so $\lambda=-\frac{1}{2} h(\xi, \xi)$. From (6.11) we conclude that $h(X, Y)=-2 \lambda g(X, Y)$, for any $X, Y \in \chi(M)$. Therefore $\mathcal{L}_{\xi} g+2 S+2 \mu \eta \otimes \eta=-2 \lambda g$. 
For $\mu=0$ follows $\mathcal{L}_{\xi} g+2 S-S(\xi, \xi) g=0$ and this gives

Corollary 6.1. On a Kenmotsu manifold $(M, \phi, \xi, \eta, g)$ with generalized symmetric metric connection with property that the symmetric $(0,2)$-tensor field $h=\mathcal{L}_{\xi} g+$ $2 S$ is parallel with respect to generalized symmetric metric connection associated to $g$, the relation (5.1), for $\mu=0$, defines a Ricci soliton.

Conversely, we shall study the consequences of the existence of $\eta$-Ricci solitons on a Kenmotsu manifold with generalized symmetric metric connection. From (6.12), we give the conclusion:

Theorem 6.3. If equation (4.9) defines an $\eta$-Ricci soliton on a Kenmotsu manifold $(M, \phi, \xi, \eta, g)$ with generalized symmetric metric connection, then $(M, g)$ is quasi-Einstein.

Recall that the manifold is called quasi-Einstein [8] if the Ricci curvature tensor field $S$ is a linear combination (with real scalars $\lambda$ and $\mu$ respectively, with $\mu \neq 0$ ) of $g$ and the tensor product of a non-zero 1-from $\eta$ satisfying $\eta=g(X, \xi)$, for $\xi$ a unit vector field and respectively, Einstein [8] if $S$ is collinear with $g$.

Theorem 6.4. If $(\phi, \xi, \eta, g)$ is a Kenmotsu structure with generalized symmetric metric connection on $M$ and (4.9) defines an $\eta$-Ricci soliton on $M$, then

1. $Q \circ \phi=\phi \circ Q$

2. $Q$ and $S$ are parallel along $\xi$.

Proof. The first statement follows from a direct computation and for the second one, note that

$$
\left(\bar{\nabla}_{\xi} Q\right) X=\bar{\nabla}_{\xi} Q X-Q\left(\bar{\nabla}_{\xi} X\right)
$$

and

$$
\left(\bar{\nabla}_{\xi} S\right)(X, Y)=\xi(S(X, Y))-S\left(\bar{\nabla}_{\xi} X, Y\right)-S\left(X, \bar{\nabla}_{\xi} Y\right) .
$$

Replacing $Q$ and $S$ from (6.14) and (6.13) we get the conclusion.

A particular case arises when the manifold is $\phi$-Ricci symmetric, which means that $\phi^{2} \circ \nabla Q=0$, as stated in the next theorem.

Theorem 6.5. Let $(M, \phi, \xi, \eta, g)$ be a Kenmotsu manifold with generalized symmetric metric connection. If $M$ is $\phi$-Ricci symmetric and (4.9) defines an $\eta$-Ricci soliton on $M$, then $\mu=1$ and $(M, g)$ is Einstein manifold [8].

Proof. Replacing $Q$ from (6.14) in (6.16) and applying $\phi^{2}$ we obtain

$$
(\alpha+1-\mu) \eta(Y)[X-\eta(X) \xi]=0,
$$

for any $X, Y \in \chi(M)$. Follows $\mu=\alpha+1$ and $S=-(\lambda+\alpha+1) g$. 
Remark 6.1. In particular, the existence of an $\eta$-Ricci soliton on a Kenmotsu manifold with generalized symmetric metric connection which is Ricci symmetric (i.e. $\bar{\nabla} S=0$ ) implies that $M$ is Einstein manifold. The class of Ricci symmetric manifold represents an extension of class of Einstein manifold to which the locally symmetric manifold also belong (i.e. satisfying $\bar{\nabla} R=0$ ). The condition $\bar{\nabla} S=0$ implies $\bar{R} \cdot \bar{S}=0$ and the manifolds satisfying this condition are called Ricci semi-symmetric [7].

In what follows we shall consider $\eta$-Ricci solitons requiring for the curvature to satisfy $\bar{R}(\xi, X) \cdot \bar{S}=0, \bar{S} \cdot \bar{R}(\xi, X)=0, \bar{W}_{2}(\xi, X) \cdot \bar{S}=0$ and $\bar{S} \cdot \bar{W}_{2}(\xi, X)=0$ respectively, where the $W_{2}$-curvature tensor field is the curvature tensor introduced by G. P. Pokhariyal and R. S. Mishra in [21]:

$$
W_{2}(X, Y) Z=R(X, Y) Z+\frac{1}{\operatorname{dim} M-1}[g(X, Z) Q Y-g(Y, Z) Q X]
$$

\section{7. $\quad \eta$-Ricci solitions on a Kenmotsu manifold with generalized symmetric metric connection satisfying $\bar{R}(\xi, X) \cdot \bar{S}=0$}

Now we consider a Kenmotsu manifold with with a generalized symmetric metric connection $\bar{\nabla}$ satisfying the condition

$$
\bar{S}(\bar{R}(\xi, X) Y, Z)+\bar{S}(Y, \bar{R}(\xi, X) Z)=0,
$$

for any $X, Y \in \chi(M)$.

Replacing the expression of $\bar{S}$ from (6.12) and from the symmetries of $\bar{R}$ we get

$$
(\alpha+1)(\alpha+1-\mu)[\eta(Y) g(X, Z)+\eta(Z) g(X, Y)-2 \eta(X) \eta(Y) \eta(Z)]=0,
$$

for any $X, Y \in \chi(M)$.

For $Z=\xi$ we have

$$
(\alpha+1)(\alpha+1-\mu) g(\phi X, \phi Y)=0
$$

for any $X, Y \in \chi(M)$.

Hence we can state the following theorem:

Theorem 7.1. If a Kenmotsu manifold with a generalized symmetric metric connection $\bar{\nabla},(g, \xi, \lambda, \mu)$ is an $\eta$-Ricci soliton on $M$ and it satisfies $\bar{R}(\xi, X) \cdot \bar{S}=0$, then the manifold is an $\eta$-Einstein manifold.

For $\mu=0$, we deduce:

Corollary 7.1. On a Kenmotsu manifold with a generalized symmetric metric connection satisfying $\bar{R}(\xi, X) \cdot \bar{S}=0$, there is no $\eta$-Ricci soliton with the potential vector field $\xi$. 


\section{8. $\eta$-Ricci solitons on Kenmotsu manifold with generalized symmetric} metric connection satisfying $\bar{S} \cdot \bar{R}(\xi, X)=0$

In this section, we have considered Kenmotsu manifold with a generalized symmetric metric connection $\bar{S}$ satisfying the condition

$$
\begin{gathered}
\bar{S}(X, \bar{R}(Y, Z) W) \xi-\bar{S}(\xi, \bar{R}(Y, Z) W) X+\bar{S}(X, Y) \bar{R}(\xi, Z) W- \\
-\bar{S}(\xi, Y) \bar{R}(X, Z) W+\bar{S}(X, Z) \bar{R}(Y, \xi) W-\bar{S}(\xi, Z) \bar{R}(Y, X) W+ \\
+\bar{S}(X, W) \bar{R}(Y, Z) \xi-\bar{S}(\xi, W) \bar{R}(Y, Z) X=0
\end{gathered}
$$

for any $X, Y, Z, W \in \chi(M)$.

Taking the inner product with $\xi$, the equation (8.1) becomes

$(8.5)-\bar{S}(\xi, Y) \eta(\bar{R}(X, Z) W)+\bar{S}(X, Z) \eta(\bar{R}(Y, \xi) W)-\bar{S}(\xi, Z) \eta(\bar{R}(Y, X) W)+$

$$
+\bar{S}(X, W) \eta(\bar{R}(Y, Z) \xi)-\bar{S}(\xi, W) \eta(\bar{R}(Y, Z) X)=0
$$

for any $X, Y, Z, W \in \chi(M)$.

For $W=\xi$, using the equation (4.4), (4.5), (4.7) and (6.12) in (8.4), we get

$(\alpha+1)(2 \lambda+\mu+\alpha+1)[g(X, Y) \eta(Z)-g(X, Z) \eta(Y)+\beta g(\phi X, Y) \eta(Z)-g(\phi X, Z) \eta(Y)]$

for any $X, Y, Z, W \in \chi(M)$.

Hence we can state the following theorem:

Theorem 8.1. If $(M, \phi, \xi, \eta, g)$ is a Kenmotsu manifold with a generalized symmetric metric connection, $(g, \xi, \lambda, \mu)$ is an $\eta$-Ricci soliton on $M$ and it satisfies $\bar{S} . \bar{R}(\xi, X)=0$. Then

$$
(\alpha+1)(2 \lambda+\mu+\alpha+1)=0 .
$$

For $\mu=0$ follows $\lambda=-\frac{\alpha+1}{2},(\alpha \neq-1)$, therefore, we have the following corollary:

Corollary 8.1. On a Kenmotsu manifold with a generalized symmetric metric connection, satisfying $\bar{S} \cdot \bar{R}(\xi, X)=0$, the Ricci soliton defined by (5.1), $\mu=0$ is either shrinking or expanding.

\section{9. $\quad \eta$-Ricci soliton on $(\varepsilon)$-Kenmotsu manifold with a semi-symmetric}

$$
\text { metric connection satisfying } \bar{W}_{2}(\xi, X) \cdot \bar{S}=0
$$

The condition that must be satisfied by $\bar{S}$ is

$$
\bar{S}\left(\bar{W}_{2}(\xi, X) Y, Z\right)+\bar{S}\left(Y, \bar{W}_{2}(\xi, X) Z\right)=0,
$$


for any $X, Y, Z \in \chi(M)$.

For $X=\xi$, using (4.4), (4.5), (4.7), (6.12) and (6.19) in (9.1), we get

$$
\frac{(\alpha+1-\mu)(-2 \mu-2 \lambda+(4 \alpha+4) n)}{n} \eta(Y) \eta(Z)
$$

for any $X, Y, Z \in \chi(M)$. Hence, we can state the following:

Theorem 9.1. If $(M, \phi, \xi, \eta, g)$ is an $(2 n+1)$-dimensional Kenmotsu manifold with a generalized symmetric metric connection, $(g, \xi, \lambda, \mu)$ is an $\eta$-Ricci soliton on $M$ and $\bar{W}_{2}(\xi, X) \cdot \bar{S}=0$, then

$$
(\alpha+1-\mu)(-2 \mu-2 \lambda+(4 \alpha+4) n)=0 .
$$

For $\mu=0$ follows that $\lambda=\frac{(4 \alpha+4) n}{2},(\alpha \neq-1)$, therefore, we have the following corollary:

Corollary 9.1. On a Kenmotsu manifold with a generalized symmetric metric connection, satisfying $\bar{W}_{2}(\xi, X) \cdot \bar{S}=0$, the Ricci soliton defined by (5.1), $\mu=0$ is either shrinking or expanding.

\section{0. $\eta$-Ricci soliton on Kenmotsu manifold with a generalized} symmetric metric connection satisfying $\bar{S} \cdot \bar{W}_{2}(\xi, X)=0$

In this section, we have considered an $(\varepsilon)$-Kenmotsu manifold with a semi-symmetric metric connection $\bar{\nabla}$ satisfying the condition

$$
\begin{gathered}
\bar{S}\left(X, \bar{W}_{2}(Y, Z) V\right) \xi-\bar{S}\left(\xi, \bar{W}_{2}(Y, Z) V\right) X+\bar{S}(X, Y) \bar{W}_{2}(\xi, Z) V- \\
-\bar{S}(\xi, Y) \bar{W}_{2}(X, Z) V+\bar{S}(X, Z) \bar{W}_{2}(Y, \xi) V-\bar{S}(\xi, Z) \bar{W}_{2}(Y, X) V+ \\
+\bar{S}(X, V) \bar{W}_{2}(Y, Z) \xi-\bar{S}(\xi, V) \bar{W}_{2}(Y, Z) X=0
\end{gathered}
$$

for any $X, Y, Z, V \in \chi(M)$.

Taking the inner product with $\xi$, the equation (10.1) becomes

$(10.5) \bar{S}(\xi, Y) \eta\left(\bar{W}_{2}(X, Z) V\right)+\bar{S}(X, Z) \eta\left(\bar{W}_{2}(Y, \xi) V\right)-\bar{S}(\xi, Z) \eta\left(\bar{W}_{2}(Y, X) V\right)+$

$$
+\bar{S}(X, V) \eta\left(\bar{W}_{2}(Y, Z) \xi\right)-\bar{S}(\xi, V) \eta\left(\bar{W}_{2}(Y, Z) X\right)=0,
$$

for any $X, Y, Z, V \in \chi(M)$.

For $X=V=\xi$, using (4.4), (4.5), (4.7), (6.12) and (6.19) in (10.4), we get

$$
(10.7)\left\{-(\alpha+1)(2 \lambda+\alpha+1+\mu)+\frac{(\lambda+\alpha+1)^{2}+(\lambda+\mu)^{2}}{2 n}\right\}\{\eta(X) \eta(Y)-g(X, Y)\}
$$

$$
+\beta(\alpha+1)(2 \lambda+\alpha+1+\mu) g(\phi X, Y)=0,
$$

for any $X, Y, Z \in \chi(M)$. Hence, we can state: 
Theorem 10.1. If $(M, \phi, \xi, \eta, g)$ is a $(2 n+1)$-dimensional Kenmotsu manifold with generalized symmetric metric connection, $(g, \xi, \lambda, \mu)$ is an $\eta$-Ricci soliton on $M$ and $\bar{S} \cdot \bar{W}_{2}(\xi, X)=0$, then

$$
-(\alpha+1)(2 \lambda+\alpha+1+\mu)+\frac{(\lambda+\alpha+1)^{2}+(\lambda+\mu)^{2}}{2 n}=0,
$$

and

$$
\beta(\alpha+1)(2 \lambda+\alpha+1+\mu)=0 .
$$

For $\mu=0$ we get the following corollary:

Corollary 10.1. On a Kenmotsu manifold with a generalized symmetric metric connection satisfying $\bar{S} \cdot \bar{W}_{2}(\xi, X)=0$, the Ricci soliton defined by (5.1), for $\mu=0$, we have the following expressions:

(i) $-(\alpha+1)(2 \lambda+\alpha+1)+\frac{(\lambda+\alpha+1)^{2}+(\lambda)^{2}}{2 n}=0$ and $\beta(\alpha+1)(2 \lambda+\alpha+1)=0$.

(ii) If $\alpha=-1$ or $\alpha=-2 \lambda-1$ which is steady.

Acknowledgement. The authors are thankful to the referee for his/her valuable comments and suggestions towards the improvement of the paper.

\section{REFEREN CES}

1. N. S. Agashe, M. R. Chafle: A semi symetric non-metric connection in a Riemannian manifold. Indian J. Pure Appl. Math. 23 (1992), 399-409.

2. A. M. BlAGA: $\eta$-Ricci solitons on Lorentzian para-Sasakian manifolds. Filomat 30 (2016), no. 2, 489-496.

3. A. M. BlaGa: $\eta$-Ricci solitons on para-Kenmotsu manifolds. Balkan J. Geom. Appl., 20 (2015), 1-13.

4. C. S. Bagewadi, G. Ingalahalli: Ricci Solitons in Lorentzian $\alpha$-Sasakian Manifolds. Acta Math. Acad. Paedagog. Nyhzi. (N.S.) 28(1) (2012), 59-68.

5. D. E.BlAIR: Contact manifolds in Riemannian geometry. Lecture note in Mathematics, 509, Springer-Verlag Berlin-New York, 1976.

6. J. T. Сhо, M. KimuRA Ricci solitons and Real hypersurfaces in a complex space form, Tohoku Math.J., 61(2009), 205-212.

7. O. Сhodosh, F. T. H. Fong: Rotational symmetry of conical Kahler-Ricci solitons. arxiv:1304.0277v2.2013,.

8. M. C. Chaki, R. K. Maity: On quasi Einstein manifolds. Publ. Math. Debrecen 57 (2000), 297-306.

9. U. C. De, D. Kamilya: Hypersurfaces of Rieamnnian manifold with semi-symmetric non-metric connection. J. Indian Inst. Sci. 75 (1995), 707-710.

10. A. Friedmann, J. A. Schouten: Uber die Geometric der halbsymmetrischen Ubertragung. Math. Z. 21 (1924), 211-223. 
11. S.Golab: On semi-symmetric and quarter-symmetric linear connections. Tensor 29 (1975), 249-254.

12. H. A. Hayden: Subspaces of space with torsion. Proc. London Math. Soc. 34 (1932), $27-50$.

13. A. Haseeb, M. A. Khan, M. D. SiddiqI: Some more Results On $\varepsilon$-Kenmotsu Manifold With a Semi-Symmetric metric connection. Acta Math. Univ. Comenianae, vol. LXXXV, 1(2016), 9-20.

14. R. S. Hamilton: The Ricci flow on surfaces. Mathematics and general relativity, (Santa Cruz. CA, 1986), Contemp. Math. 71, Amer. Math. Soc., (1988), 237-262.

15. J. B. Jun, U. C. De, G. Pathak: On Kenmotsu manifolds. J. Korean Math. Soc. 42 (2005), no. 3, 435-445.

16. H. LEVY: Symmetric tensors of the second order whose covariant derivatives vanish, Ann. Math. 27(2) (1925), 91-98.

17. H. G. Nagaraja, C. R. Premalatha: Ricci solitons in Kenmotsu manifolds. J. Math. Anal. 3 (2) (2012), 18-24.

18. K. Kenmotsu: A class of almost contact Riemannian manifold. Tohoku Math. J., 24 (1972), 93-103.

19. G. PATHAK, U. C. DE: On a semi-symmetric connection in a Kenmotsu manifold. Bull. Calcutta Math. Soc. 94 (2002), no. 4, 319-324.

20. D. G. Prakasha, B. S. HADIMANi: $\eta$-Ricci solitons on para-Sasakian manifolds. J. Geom., DOI 10.1007/s00022-016-0345-z.

21. G. P. POKhARIYAL, R. S. Mishra: The curvature tensors and their relativistic significance. Yokohama Math. J. 18 (1970), 105-108.

22. R. Sharma: Certain results on $K$-contact and $(k, \mu)$-contact manifolds. J. Geom., 89(1-2) (2008), 138-147.

23. A. Sharfuddin, S. I. Hussain: Semi-symmetric metric connections in almost contact manifolds. Tensor (N.S.), 30(1976), 133-139.

24. B. G. SChmidt: Conditions on a connection to be a metric connection. Commun. Math. Phys. 29 (1973), 55-59.

25. M. D. SiddiqI, M. Ahmad, J. P. OJHA: CR-submanifolds of a nearly trans-hyperpolic Sasakian manifold with semi-symmetric non metric connection. African. Diaspora J. Math. (N.S.) 2014, 17 (1), 93-105

26. M. M. TRIPATHI: On a semi-symmetric metric connection in a Kenmotsu manifold, J. Pure Math. 16(1999), 67-71.

27. M. M. TRIPATHI, N. NAKKAR : On a semi-symmetric non-metric connection in a Kenmotsu manifold. Bull. Cal. Math. Soc. 16 (2001), no.4, 323-330.

28. M. M. TRIPATHI: Ricci solitons in contact metric manifolds. arXiv:0801.4222 [math.DG].

29. K. Yano: On semi-symmetric metric connections. Revue Roumaine De Math. Pures Appl. 15(1970), 1579-1586.

30. K. Yano, M. Kon: Structures on Manifolds. Series in Pure Math., Vol. 3, World Sci., 1984. 
Mohd Danish Siddiqi

College of Science

Department of Mathematics

Jazan University

Jazan, Kingdom of Saudi Arabia.

anallintegral@gmail.com, msiddiqi@jazanu.edu.sa

Oğuzhan Bahadır

Faculty of Science and Letters

Department of Mathematics

Kahramanmaras Sutcu Imam University,

Kahramanmaras, TURKEY

oguzbaha@gmail.com 